\title{
First molecular detection of porcine bocavirus in Malaysia
}

\begin{abstract}
Several strains of porcine bocaviruses have been reported worldwide since their first detection in Sweden in 2009. Subsequently, the virus has been reported to be associated with gastrointestinal and respiratory signs in weaner and grower pigs. Although Malaysia is host to a self-sufficient swine livestock industry, there is no study that describes porcine bocavirus in the country. This report is the first to describe porcine bocavirus (PBoV) in Malaysian swine herds. $\mathrm{PBoV}$ was identified in various tissues from sick and runt pigs using the conventional PCR method with primers targeting conserved regions encoding for the nonstructural protein (NS1) gene. Out of 103 samples tested from 17 pigs, 32 samples from 15 pigs were positive for porcine bocavirus. In addition, a higher detection rate was identified from mesenteric lymph nodes $(52.9 \%)$, followed by tonsil (37.0\%), and lungs (33.3\%). Pairwise comparison and phylogenetic analyses based on a 658-bp fragment of NS1 gene revealed that the Malaysian $\mathrm{PBoV}$ strains are highly similar to PBoV3 isolated in Minnesota, USA. The presence of porcine bocavirus in Malaysia and their phylogenetic bond was marked for the first time by this study. Further studies will establish the molecular epidemiology of PBoV in Malaysia and clarify pathogenicity of the local isolates.
\end{abstract}

Keyword: Porcine bocavirus; Swine; Detection; Sequencing; Phylogenetic analysis 
